\title{
SUR L'UTILISATION DE LA POUDRE DE LAIT DANS L'ÉLEVAGE des PRÉmATURÉS DÉbILES
}

\author{
par le Dr. ROUME \\ Ancien Chef de Clinique Obstétricale à la Faeulté de Montpellier
}

II y a quelques mois, j'étais appelé auprès d'une jeune femme enceinte de six mois et demi environ, elle était dans un état de santé fort précaire, atteinte d'une pleurite de la base. En quelques jours son état s'était aggravé et le travail s'était déclaré prématurément. Dans ces conditions, je ne tentais rien pour arrêter l'accouchement; au contraire, je décidais de le terminer artificiellement dans l'intérêt de la mère, ne comptant pas sur la survie d'un enfant déjà bien compromis.

Après dilatation artificielle du col, la version podalique me.permit d'extraire le foetus, toutes les manœuvres furent conduites sous anesthésie générale avec un minimum de traumatisme. L'enfant était de petite taille, mais bien conformé. Né en état d'apné, il fut ranimé non sans peine par les procédés habituels. Je considérais ce débile comme voué à une mort prochaine, cependant je lui fis donner les soins nécessaires.

Il pesait $1.450 \mathrm{gr}$.

Le lendemain, à ma surprise, je trouvais cet enfant bien vivant, éveillé; il piaulait faiblement. Pour le sauver, il fallait le défendre contre les deux redoutables ennemis des prématurés : le refroidissement et l'inanition.

Comment nourrir ce prématuré débile auquel sa mère ne pouvait donner le sein et pour lequel il ne pouvait être question de trouver une nourrice? Sachant combien l'allaitement artificiel est difficile chez ces enfants aux fonctions digestives insuffisantes, je pensais au lait sec, dont la grande digestibilité et la bonne utilisation physiologique pouvaient nous donner quelques chances de succès.

L'enfant fut donc placé en couveuse, celle-ci réglée soigneusement aux alentours de 28 degrés. Toutes les deux heures on le sortait avec précaution et on s'efforçait de lui faire prendre deux cuillerées à eafé soit dix grammes du mélange suivant:

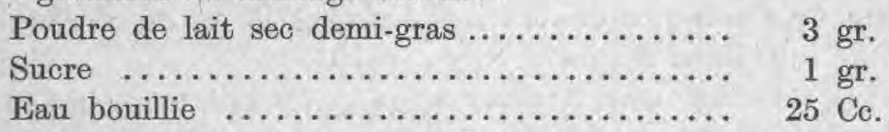

Ce mélange représentait environ 700 calories pour $1,000 \mathrm{Cc}$., chiffre identique à la valeur d'un lait de femme de composition moyenne.

Avec beaucoup de patience, la garde réussit à faire ingérer ainsi $60 \mathrm{cc}$. de ce mélange le second jour, et autant le troisième, mais finalement l'enfant ne voulant ni sucer à la cuiller, ni têter, il fallutle gaver; de cette façon nous pûmes arriver à lui faire prendre des doses croissantes.

Dans les jours suivants, sans changer la composition de ce lait, 
nous avons essayé d'atteindre les doses journalières que BuDIN avait établies pour le lait maternel. Dans cetableau nous répétons les chiffres de BuDIN et inscrivons à côté ce que nous avons donné à notre avorton.

\begin{tabular}{|c|c|c|}
\hline Enfan & $\begin{array}{l}\text { nts de moins de } 1800 \mathrm{gr} \text {. } \\
\text { Chiffres de BuDIN } \\
\text { (lait maternel) }\end{array}$ & $\begin{array}{l}\text { Quantité ingérée par } \\
\text { notre prématuré } \\
\text { (lait reconstitué) }\end{array}$ \\
\hline $1 \ldots \ldots \ldots \ldots \ldots \ldots \ldots$ & & néant \\
\hline $2 \ldots \ldots \ldots \ldots \ldots \ldots \ldots$ & 115 & 60 \\
\hline $3 \ldots \ldots \ldots \ldots$ & 160 & 60 \\
\hline $4 \ldots \ldots \ldots \ldots \ldots \ldots \ldots \ldots$ & 210 & 120 \\
\hline $5 \ldots \ldots \ldots \ldots \ldots \ldots \ldots$ & 225 & 200 \\
\hline $6 \ldots \ldots \ldots \ldots$ & 250 & 225 \\
\hline $7 \ldots \ldots \ldots \ldots$ & 280 & 250 \\
\hline $8 \ldots \ldots \ldots \ldots$ & 285 & 280 \\
\hline $9 \ldots \ldots \ldots \ldots$ & 310 & 300 \\
\hline $10 \ldots \ldots \ldots \ldots \ldots \ldots \ldots$ & 320 & 300 \\
\hline
\end{tabular}

Le résultat dépassa notre attente. Au $10^{\mathrm{e}}$ jour l'enfant avait augmenté notablement de poids, sa température rectale qui était le lendemain de sa naissance de 35,9 et qui le $5^{\mathrm{e}}$ jour était descendue à 35,4 était remontée et atteignait 36,8 .

A partir du $10^{\mathrm{e}}$ jour, l'enfant toujours en couveuse fut réglé très régulièrement: dix prises d'aliment par jour qu'il se mit à prendre à la cuiller. Le mélange fut modifié de façon à augmenter sa valeur calorigène sans changer beaucoup de volume.

A la poudre de lait demi-écrémé, je fis substituer la poudre de lait entier, la quantité de poudre et de sucre fut augmentée prudemment et nous fûmes ainsi conduits à utiliser des mélanges correspondant d'abord à 700 calories par 1.000 cc., puis à 766 calories, puis à 915 et 970, pour redescendre ensuite et nous rapprocher d'un aliment de composition normale à mesure que l'enfant grossissait.

Durant les deux premiers mois, l'augmentation fut très régulière de 30 gr. par jour, puis durant les deux seconds elle fut encore de 25 et 22 gr. par jour.

A l'âge d'un mois, cet enfant pesait 2.250 gr. et consommait par 24 heures, $60 \mathrm{gr}$. de poudre de lait complet et $15 \mathrm{gr}$. de sucre (environ 400 cc. de liquide en 9 prises).

A l'âge de deux mois, il pesait 3.100 gr. et consommait par $24 \mathrm{~h}$. $70 \mathrm{gr}$. de poudre de lait complet et $20 \mathrm{gr}$. de sucre (environ $450 \mathrm{cc}$. de liquide en 9 prises).

A l'âge de trois mois, il pesait 3.800 gr. et consommait par $24 \mathbf{h}$. $80 \mathrm{gr}$. de poudre de lait complet et $30 \mathrm{gr}$. de sucre (environ $640 \mathrm{cc}$. de liquide).

Dans la seconde moitié du quatrième mois, l'enfant atteignait 4.200 gr.; il consommait par 24 heures 90 gr. de poudre de lait, son état général 


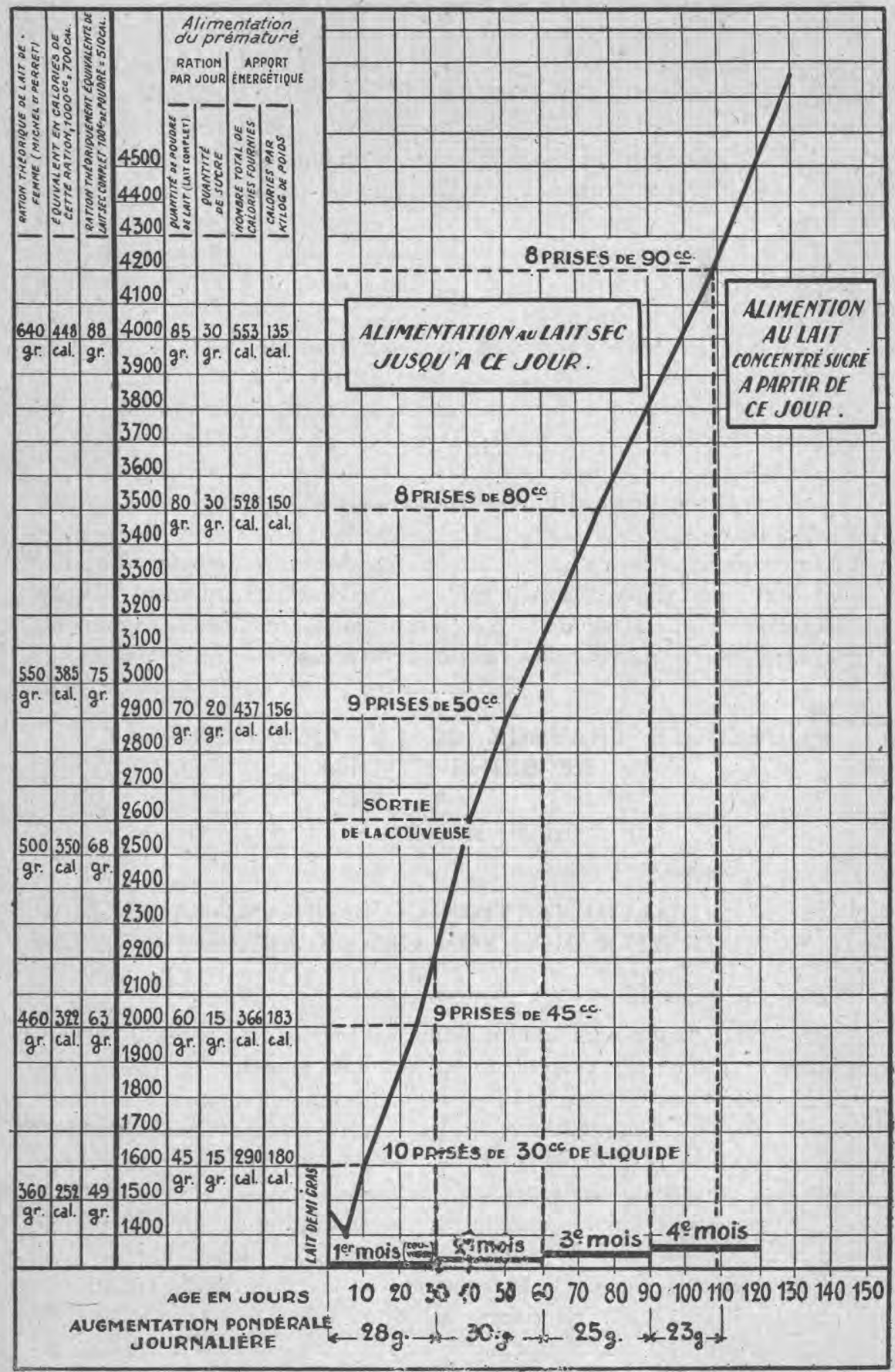


était excellent, on aurait dit un bébé au sein. L'alimentation au lait sec devenait très onéreuse, aussi les parents ont demandé à la modifier. Nous essayâmes alors le lait de vache sous la forme de lait condensé sucré, le bébé s'y adapta très facilement, sa courbe de poids ne subit aucune perturbation et, depuis, sa croissance a continué sans incident.

C'est aujourd'hui un beau bébé de sept mois qui fait honneur à l'infirmière qui s'est attachée à lui et qui n'a ménagéni son temps ni sa peine.

A noter que depuis le début du troisième mois, je lui faisais donner chaque jour une cuiller à café de jus de fruit frais pour apporter des vitamines,

L'élevage des prématurés est une question de soins minutieux. La mortalité chez eux est considérable. Budin qui, par ses travaux, a contribué beaueoup à perfectionner leur élevage, trouvait $85 \%$ de mortalité chez les enfants de 1.200 à 1.500 gr. et cependant dans un service hospitalier spécialisé avec un personnel éduqué et la possibilité d'utiliser le lait de femme! Plus tard, dans les mêmes conditions, TARNIER accusait $60 \%$.

Notre observation n'a pas d'autre but que de montrer (ce que d'autres ont fait avant nous) que le lait sec est parfaitement supporté par ces petits êtres et que le lait de la mère étant déficient, c'est là un aliment de remplacement qui peut être utilisé avec suceès.

\section{QUELQUES TRAVAUX DE L'OFFICE AGRICOLE DE SEINE-ET-OISE}

(fin)

par M. MONMIREL,

Président de l'Office agricole départemental de Seine-et-Oise

\section{ESSAIS D'ALIMENTATION GONGERNANT LA VALEUR NUTRITIVE D'UN SON GRAS D'ARACHIDE}

\section{1. - But des essais.}

Sur le marché des aliments du bétail, les tourteaux d'arachide extrablancs font prime, car l'aspect de ces produits constitue généralement une garantie d'excellente qualité. Pour obtenir de pareils produits, certains usiniers éliminent avec le plus grand soin avant le passage dans les presses la pellicule externe, de couleur rose, des arachides préalablement privées de leurs coques fibreuses. Ce travail s'exécute avec un matériel spécial et donne comme sous produit un son d'arachide dont l'odeur rappelle celle du tourteau, gras au toucher, et qui, normalement, se compose de la pellicule rose mélangée à des fragments d'amande, à l'exclusion de débris de coque.

L'attention de quelques membres du syndicat des éleveurs de Seine- 